\title{
Modelos analíticos do crescimento populacional de Sitophilus zeamais em trigo armazenado
}

\author{
Arienilmar A. L. da Silva', Lêda R. D’A. Faroni², Raul N. C. Guedes², José H. Martins² \& Marco A. G. Pimentel ${ }^{2}$
}

\begin{abstract}
RESUMO
Para se atingir os objetivos propostos neste trabalho, desenvolveram-se modelos analíticos do crescimento populacional de $S$. zeamais em grãos de trigo armazenados com teor de umidade de $11,1 \%$. O crescimento populacional foi avaliado durante 90 dias de armazenagem, nas temperaturas de $16,20,24,28,32$ e $36{ }^{\circ} \mathrm{C}$, partindo-se de infestações iniciais que variaram de 1,3 a 9,3 insetos por kg de trigo. Os modelos explicaram de 93,9 a 98,9\% da variação na densidade populacional. O crescimento populacional foi afetado pelo tamanho da população que iniciou a infestação, ou seja, quanto maior foi a infestação inicial maior também foi o crescimento populacional ao longo do tempo de armazenagem, que ocorreu na temperatura de $28{ }^{\circ} \mathrm{C}$ e diminuiu drasticamente abaixo e acima desta. Na temperatura de $36{ }^{\circ} \mathrm{C}$, todas as populações foram extintas entre 15 e 45 dias de armazenagem, mas nenhuma população se extinguiu a $16^{\circ} \mathrm{C}$. Os modelos apresentaram excelentes ajustes, estando em conformidade com os princípios gerais da dinâmica de populações de insetos em um sistema de grãos armazenados e descrevem muito bem o crescimento populacional de $S$. zeamais em relação ao efeito da temperatura e da densidade populacional inicial.
\end{abstract}

Palavras-chave: grãos, insetos, infestação, temperatura

\section{Analytical models for population growth of Sitophilus zeamais in stored wheat}

\begin{abstract}
Analytical models of population growth were developed for Sitophilus zeamais, in grain wheat stored at $11.1 \%$ of moisture content. Population growth was monitored for 90 days at the temperatures of $16,20,24,28,32$ and $36{ }^{\circ} \mathrm{C}$ and initial adult population densities varying between 1.3 and 9.3 insects per $\mathrm{kg}$. The models obtained explained between 93.9 and $98.99 \%$ of the variation in density of adult insects. The population growth was affected by initial population density of infestation and increased proportionately with degree of infestation. The highest population growth occurred at $28{ }^{\circ} \mathrm{C}$ and drastically decreased for lower and higher temperatures. Population extinction took place between 15 and 45 days of storage for all samples maintained at $36{ }^{\circ} \mathrm{C}$, but no extinction was observed at $16{ }^{\circ} \mathrm{C}$. The models obtained present excellent adjustments and are in agreement with general principles underlying the pest-storage system; they reproduce very well the general effect of temperature and initial population densities upon S. zeamais population growth.
\end{abstract}

Key words: grains, insects, infestation, temperature 


\section{INTRODUÇÃO}

Sitophilus zeamais (Motschulsky) (Coleoptera: Curculionidae) praga cosmopolita de cereais armazenados (Throne, 1994) sujeita ao uso intenso de inseticidas, especialmente em áreas tropicais, devido à carência de métodos de controle alternativos eficientes (Guedes et al., 1995, 1996); entretanto, a crescente pressão sobre as indústrias de grãos para reduzirem sua dependência ao uso de inseticidas, tem levado à adoção do manejo integrado de pragas que, por sua vez, pode ser auxiliado pelas predições realizadas por modelos de crescimento populacional de insetos. Os modelos matemáticos são ferramentas importantes para predizer, avaliar e compreender a dinâmica de populações de pragas em ecossistemas naturais ou criados pelo homem em uma variedade de condições ambientais e de ações de manejo (Smerage, 1992). Com esses modelos, diferentes estratégias de controle podem ser comparadas. Os modelos são especialmente úteis para se predizer a eficiência de métodos não químicos de controle em grãos armazenados (Hagstrum \& Flinn, 1996).

Muitos tipos de modelo são usados no estudo e no manejo de insetos, tais como: modelos de regressão (estatísticos), de período de infecção, fisiológicos $\left({ }^{\circ} \mathrm{d}^{-1}\right)$, para simulação, analíticos e para otimização (Norton et al., 1993; Berryman, 1997). Se o sistema em estudo é controlado e as relações que compõem seu modelo são simples o bastante, é possível trabalhar com elas, de modo a se conseguir uma solução analítica; neste caso, tal modelo é chamado modelo analítico, porém muitos sistemas são altamente complexos e seus modelos matemáticos válidos também o são, excluindo qualquer possibilidade de uma solução analítica; desta forma, o modelo deve ser estudado por meio de simulação, ou seja, exercitá-lo numericamente com dados de entrada de interesse para observar como eles afetam as medidas de performance dos dados de saída (Law \& Kelton, 1991). No estudo de insetos, os modelos para simulação visam predições exatas e detalhadas sobre a dinâmica populacional de sistemas particulares, enquanto os modelos analíticos desconsideram os detalhes e, em vez disso, visam capturar a essência da dinâmica populacional de um sistema e seus princípios gerais, de maneira qualitativa, mas ambos podem ser usados para se complementarem um ao outro (Hess, 1996). A maioria das pesquisas visa aos modelos de simulação, capazes de lidar com um número grande de variáveis, na busca de descrever melhor o sistema de grãos armazenados, mas mesmo esses modelos têm sido incompletos. Berryman (1997) defende a importância de se considerar, na modelagem, os quatro princípios fundamentais da teoria da dinâmica de populações: crescimento geométrico; cooperação intraespecífica; competição intraespecífica e realimentação negativa induzida pelo retardamento da densidade, devido à presença de predadores e outros inimigos naturais. A maioria desses princípios tem sido desconsiderada, mesmo nos modelos de simulação, pois as espécies são estudadas isoladamente e, na realidade, geralmente várias espécies infestam os grãos ao mesmo tempo. Portanto, de certa forma esses modelos também são apenas analíticos.
A temperatura do ar ambiente, que é o ar intergranular no caso de um sistema de grãos armazenados, e a umidade do grão, são os fatores físicos importantes no estabelecimento e desenvolvimento de uma população de insetos pragas de grãos armazenados (Kawamoto et al., 1992; Hagstrum \& Throne, 1989; Hagstrum \& Flinn, 1992). Considerando-se o sistema formado por grãos armazenados, trata-se de um sistema altamente controlado. Os grãos são armazenados depois de expurgados e com teor de umidade conhecido, estipulado na secagem, em uma estrutura projetada para isolálos ao máximo da influência externa de fatores físicos e biológicos. O próprio grão é um isolante térmico. Por tudo isso, as características psicrométricas da atmosfera intergranular são conhecidas e pouco variáveis e, conseqüentemente, a umidade do grão também é ainda menos variável. Para este sistema, pode-se assumir condições Malthusianas, isto é, o crescimento populacional é geométrico porque não há, praticamente, limitações de alimento, de espaço nem climáticas (Hardman, 1976; Throne, 1995). Portanto, é possível o uso de modelos analíticos de crescimento populacional de insetos de grãos armazenados para estudos de situações isoladas: uma só espécie de grão, umidade de grão e inseto. Entretanto, os modelos mais usados são os de simulação pois permitem avaliar simultaneamente diferentes combinações de grão, umidade, temperatura e inseto.

O crescimento populacional dos principais insetos de grãos armazenados já foi amplamente estudado e modelado na América do Norte, na Europa e na Austrália. O problema é que se trata de modelos de simulação a serem ainda disponibilizados comercialmente na forma de programas para computador. Por isso, o presente estudo objetivou desenvolver modelos analíticos de crescimento populacional do $S$. zeamais em trigo armazenado, que pudessem ser prontamente utilizados pelos pesquisadores brasileiros como ferramenta auxiliar na avaliação dos resultados de outras pesquisas relacionadas ao controle dessa praga, modelos estes que possam descrever o crescimento populacional em função do tempo e da temperatura de armazenagem, fatores físicos e, também, em função da densidade populacional que inicia a infestação, fator biológico.

\section{MATERIAL E MÉTODOS}

Utilizou-se um delineamento experimental inteiramente casualizado, em esquema fatorial 8 × 7 x 6, com oito níveis iniciais de infestação, sete períodos de armazenagem e seis condições climáticas e três repetições. As unidades experimentais consistiram de potes de vidro com $1,5 \mathrm{~kg}$ de trigo com teor de umidade de $11,1 \%$, base úmida. Para simular diferentes níveis de infestação inicial, os potes foram infestados com populações de 0,2 , 4, 6, 8, 10, 12 e 14 insetos adultos da espécie $S$. zeamais, o que representa densidades populacionais de 0,$0 ; 1,3 ; 2,7 ; 4,0 ; 5,3 ; 6,7 ; 8,0$ e 9,3 insetos por kg de trigo. Os potes foram armazenados em câmaras climáticas, onde o crescimento populacional ocorreu nas seguintes combinações: $16^{\circ} \mathrm{C}$ e $63 \%, 20^{\circ} \mathrm{C}$ e $65 \%, 24{ }^{\circ} \mathrm{C}$ e $67 \%, 28^{\circ} \mathrm{C}$ e $68,5 \%, 32{ }^{\circ} \mathrm{C}$ e $70 \%$, e $36^{\circ} \mathrm{C}$ e $71 \%$. Nessas 
combinações, se ocorresse reumedecimento, seria o mesmo e os grãos de todos os tratamentos poderiam atingir a umidade máxima de $13 \%$ e, por outro lado, esta variação na umidade relativa não é significativa para o crescimento populacional (Kawamoto et al., 1992; Hagstrum \& Throne, 1989; Hagstrum \& Flinn, 1992). Adotou-se este intervalo de temperaturas porque ele representa as temperaturas predominantes no clima brasileiro; além disso, contém a faixa de temperaturas ótimas para o desenvolvimento da espécie $S$. zeamais, 28 a $30^{\circ} \mathrm{C}$ (Hwang et al., 1983; Throne, 1994). Para a pesquisa de diferentes períodos de armazenagem, os potes infestados foram armazenados por 15, 30, 45, 60, 75 e 90 dias. Ao final de cada período, a massa de grãos de cada unidade experimental foi peneirada e o número de insetos adultos vivos foi contado e registrado. Para a contagem das populações grandes, com milhares de insetos, adotou-se o seguinte método: para cada pote pesou-se a população peneirada e, depois, se lhe tirou uma amostra, a qual foi pesada e teve seus insetos vivos contados; em seguida, a relação número de insetos por grama de amostra foi usada para se calcular a população total.

A umidade de grãos praticamente não varia ao longo de uma armazenagem adequada, sobretudo no que diz respeito ao reumedecimento, que seria o tipo de variação favorável ao desenvolvimento dos insetos. Por isso, a umidade do grão não precisa ser considerada uma variável dentro de um modelo analítico de crescimento populacional de insetos de grãos armazenados, porque ela realmente não varia o bastante para significar o estabelecimento ou não de uma população de insetos; portanto, a umidade do grão foi considerada uma constante, um dado de entrada para o modelo pois, uma vez armazenado com aquela umidade, o grão está com sua vulnerabilidade permanentemente definida.

Os modelos foram desenvolvidos realizando-se análise de regressão dos dados de população final de insetos como função do tempo e da temperatura de armazenagem, em cada nível de infestação inicial. Também foram desenvolvidos modelos com a população final de insetos, em função do tempo de armazenagem e do nível de infestação inicial, para cada temperatura. Dadas as condições Malthusianas, presumiu-se que ocorreria um crescimento geométrico e foram utilizados modelos exponenciais. Para o caso em que a quantidade de $1,5 \mathrm{~kg}$ de trigo poderia tornar-se insuficiente para alimentar a população, utilizaram-se modelos logísticos (Norton et al.,
1993; Berryman, 1997). Considerando-se apenas o efeito da temperatura, o crescimento populacional diminui acima e abaixo da temperatura ótima, o que pode ser descrito por uma parábola. Esses modelos e suas combinações foram usados como modelos de partida para a análise de regressão nos programas Statistica for Windows (Statisoft, 1993) e SigmaPlot for Windows (SPSS, 1997).

\section{RESULTADOS E DISCUSSÃO}

\section{Crescimento em função do tempo e da temperatura de armazenamento}

Modelou-se o crescimento populacional de S. zeamais em função do tempo e da temperatura de armazenamento, em cada nível de infestação inicial. Um mesmo comportamento se repetiu em todos os níveis de infestação inicial estudados, motivo pelo qual se ajustaram modelos semelhantes para todos que puderam ser descritos por uma única equação geral (Eq. 1):

$$
\mathrm{Z}=\mathrm{P}_{0}+\exp \left[\mathrm{a}+\mathrm{b} \cdot \ln (\mathrm{X})+\frac{\mathrm{c}}{\mathrm{X}}+\frac{\mathrm{d} \cdot \ln (\mathrm{X})}{\mathrm{X}^{\mathrm{e}}}+\mathrm{f} \cdot \mathrm{X}^{\mathrm{g}} \cdot \mathrm{Y}^{\mathrm{h}}\right]
$$

em que $\mathrm{Z}$ é a população final de insetos vivos predita, pelo modelo (insetos por kg de trigo); $\mathrm{P}_{0}$ é a infestação inicial (insetos por kg de trigo); $\mathrm{X}$ é a temperatura $\left({ }^{\circ} \mathrm{C}\right)$; Y é o tempo de armazenamento (dias); a, b, c, d, e, f e g, são coeficientes que variam com a infestação inicial, conforme os dados da Tabela 1.

Todos os modelos obtidos foram altamente significativos ( $<<0,0001$ com 118 Graus de Liberdade do Resíduo), explicando mais de $94 \%$ da variação ocorrida nas populações. Os gráficos A e B da Figura 1 mostram o comportamento de populações de $S$. zeamais que se desenvolveram a partir de infestações iniciais de 1,3 e 9,3 insetos por kg, respectivamente. O maior crescimento populacional, conforme os dados observados, ocorreu a $28{ }^{\circ} \mathrm{C}$ e o modelo projetou o ponto de máximo também nesta temperatura, o que está de acordo com as condições ótimas, de 28 a $30^{\circ} \mathrm{C}$, determinadas para a espécie $S$. zeamais em trabalhos, como os de Hwang et al. (1983) e Throne (1994). Fora dessa temperatura ótima, observaram-se crescimentos muito menores, ou

Tabela 1. Coeficientes e estatísticas da Equação 1 para cada infestação inicial de S. zeamais

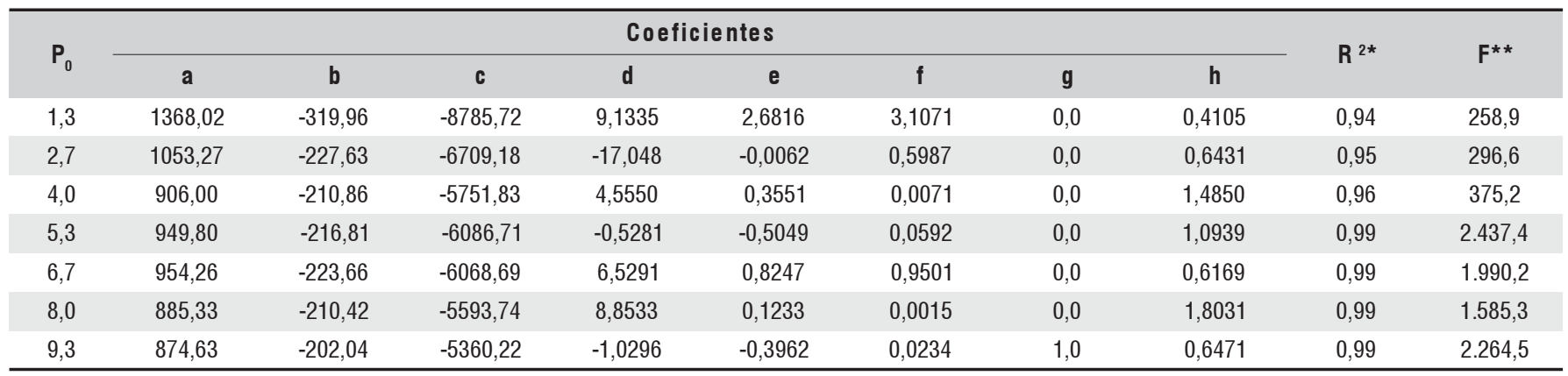

* Coeficiente de determinação múltipla;

** Quociente entre Quadrado Médio da Regressão e o Quadrado Médio do Resíduo 


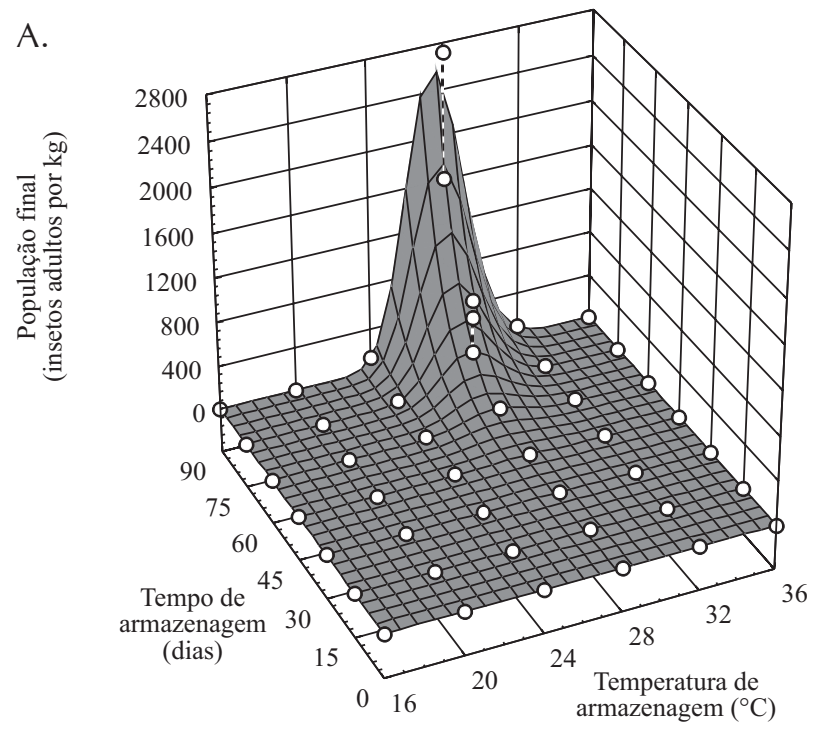

B.

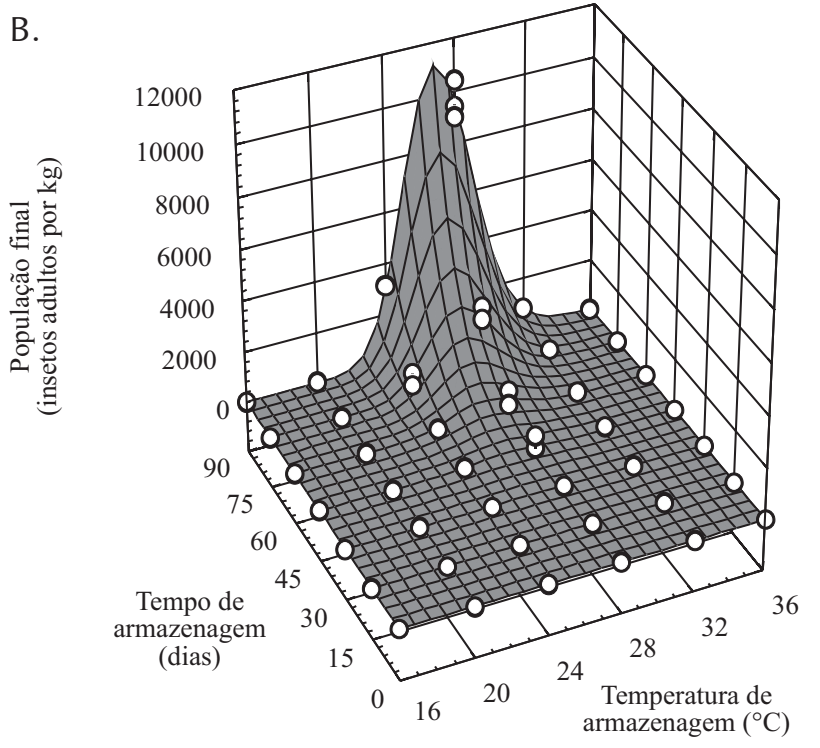

Figura 1. Valores observados (círculos) e preditos (superfície de resposta) para o crescimento populacional de $S$. zeamais a partir da infestação inicial de 1,3 (gráfico A) e 9,3 (gráfico B) insetos por kg de trigo

seja, o crescimento populacional diminuiu drasticamente em temperaturas não ótimas. No pico de crescimento em 90 dias de armazenagem, a $28^{\circ} \mathrm{C}$, a população inicial de 1,3 inseto adulto por quilograma de trigo atingiu 2.430,8 insetos
(Eq. 1). Este pico aumentou com o nível de infestação inicial, atingindo 9.603,4 insetos por quilograma de trigo a partir de 9,3 insetos, quase quatro vezes mais.

Os modelos baseados na Eq. 1 falharam em descrever o comportamento populacional observado nas temperaturas extremas de 16 e $36{ }^{\circ} \mathrm{C}$. Em $16^{\circ} \mathrm{C}$ observou-se pequeno declínio no crescimento populacional e, em $36^{\circ} \mathrm{C}$, constatouse extinção da população entre 15 e 45 dias de armazenagem, mas os modelos predisseram uma população constante e um pequeno crescimento, respectivamente. Esses comportamentos não estão visíveis nos gráficos da Figura 1 devido à grande amplitude de valores do eixo $\mathrm{z}$.

\section{Crescimento populacional em função do tempo e do nível de infestação inicial}

Modelou-se o crescimento populacional em função do tempo de armazenamento e do nível de infestação inicial, em cada temperatura. Os modelos têm a forma geral da Eq. 2, cujos coeficientes, em cada temperatura, estão na Tabela 2.

$$
\mathrm{Z}=\mathrm{Y} \cdot\left[1+\mathrm{aX} \cdot \exp (\mathrm{bX}) \cdot \mathrm{Y}^{\mathrm{c}-1}+\mathrm{dX}^{\mathrm{e}}\right]
$$

em que $\mathrm{Z}$ é a população final de insetos vivos predita pelo modelo (insetos por kg de trigo); Y é a infestação inicial (insetos por kg de trigo); $\mathrm{X}$ é o tempo de armazenamento (dias); a, b, c, d e e, são coeficientes que variam com a temperatura.

Esses modelos também foram altamente significativos ( $<<0,0001$ e 165 Graus de Liberdade do Resíduo para todos os modelos) e apresentaram coeficientes de determinação múltipla $\left(\mathrm{R}^{2}\right)$ acima de 0,93 . Em cada temperatura estudada o pico de crescimento populacional ocorreu sempre para combinação de maior infestação inicial, 9,3 insetos por kg, e maior tempo de armazenamento, 90 dias.

O efeito da infestação inicial no crescimento da população de $S$. zeamais é ilustrado nas condições desfavoráveis de $16^{\circ} \mathrm{C}$, Figura 2A, nas condições ótimas de $28^{\circ} \mathrm{C}$, Figura $2 \mathrm{~B}$, e nas condições desfavoráveis de $36^{\circ} \mathrm{C}$, Figura 2C.

Observa-se, na Figura 2A, que a população de S. zeamais decresceu lentamente sob uma temperatura de armazenagem de $16{ }^{\circ} \mathrm{C}$, em todos os níveis de infestação inicial estudados.

Tabela 2. Coeficientes da Equação 2 para cada temperatura

\begin{tabular}{|c|c|c|c|c|c|c|c|}
\hline \multirow{2}{*}{$\mathrm{T}\left({ }^{\circ} \mathrm{C}\right)$} & \multicolumn{5}{|c|}{ Coeficientes } & \multirow{2}{*}{$\mathbf{R}^{2}$ * } & \multirow{2}{*}{$F^{* *}$} \\
\hline & a & b & c & d & e & & \\
\hline 16 & $-0,0033820$ & 0,0000 & 1,0000 & $-0,00001$ & 2,0000 & 0,96 & \\
\hline 20 & 0,0000003 & 0,0984 & 1,8383 & 0,00000 & 1,0000 & 0,93 & 1166,9 \\
\hline 24 & 0,0001331 & 0,0926 & 1,8319 & 0,00000 & 1,0000 & 0,98 & 4950,5 \\
\hline 28 & 0,0089993 & 0,0819 & 0,8982 & 0,00000 & 1,0000 & 0,98 & 4541,8 \\
\hline 32 & 0,0424382 & 0,0250 & 1,3003 & 0,00000 & 1,0000 & 0,96 & 1845,63 \\
\hline 36 & 0,0035030 & 0,0000 & 1,0000 & $-0,58105$ & 0,1806 & 0,99 & 7372,8 \\
\hline
\end{tabular}

* Coeficiente de determinação múltipla;

** Quociente entre Quadrado Médio da Regressão e o Quadrado Médio do Resíduo 

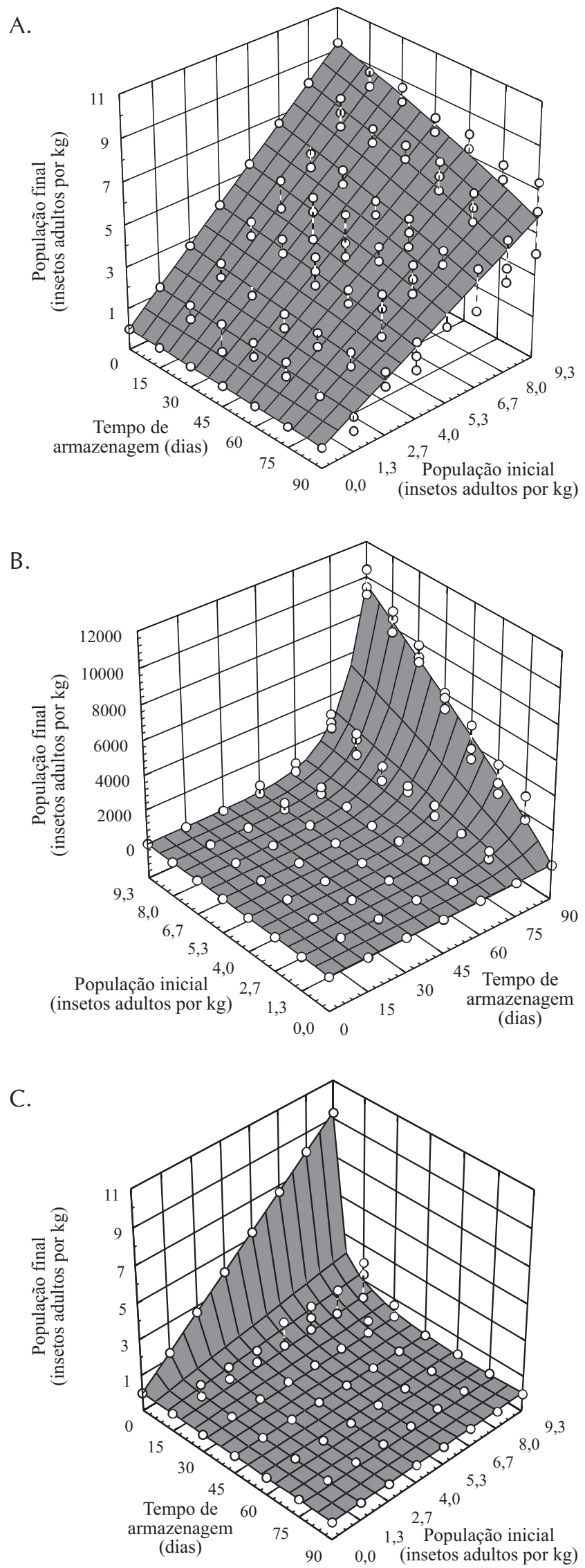

Figura 2. Valores observados (círculos) e preditos (superfície de resposta) para o crescimento populacional de $S$. zeamais a partir de níveis de infestação inicial de 0 a 9,3 insetos por kg de trigo, em temperatura de $16{ }^{\circ} \mathrm{C}(\mathrm{A}), 28^{\circ} \mathrm{C}(\mathrm{B})$ e $36{ }^{\circ} \mathrm{C}(\mathrm{C})$
Throne (1994) também notou que, abaixo de $20^{\circ} \mathrm{C}$, as fêmeas de $S$. zeamais colocam poucos ovos. A partir da temperatura de armazenagem de 20 até $32^{\circ} \mathrm{C}$, observou-se um crescimento exponencial em todos os níveis de infestação inicial, Figura $2 \mathrm{~B}$, indicando que a quantidade de alimento não foi um fator limitante nos níveis de infestação estudados. Na temperatura de $36{ }^{\circ} \mathrm{C}$, Figura $2 \mathrm{C}$, todas as populações foram extintas. A população iniciada a partir de 1,3 inseto por kg de trigo foi extinta entre 15 e 30 dias de armazenagem, e a iniciada a partir de 9,3 insetos por kg de trigo, entre 30 e 45 dias de armazenagem. Throne (1994) estudando S. zeamais, também observou que nenhum inseto adulto sobreviveu sob $35^{\circ} \mathrm{C}$. O modelo não prediz valores de populações absolutamente iguais a zero nos períodos citados mas, sim, valores infinitamente pequenos ou negativos, o que equivale à conclusão prática de extermínio acima de 45 dias de armazenagem.

Pelos resultados apresentados verifica-se o quanto é significativo o tamanho da infestação inicial para o desenvolvimento das populações. Numa pequena faixa que variou de apenas de 1,3 a 9,3 insetos por kg, tem-se crescimentos populacionais que representariam conseqüências práticas diferentes para o controle da praga.

São comuns o desenvolvimento e a aplicação de modelos analíticos no manejo de pragas de lavouras (Norton et al., 1993; Hess, 1996; Berryman, 1997). Para pragas de grãos armazenados predomina o desenvolvimento de modelos de simulação, mas os resultados de modelos analíticos e de simulação podem ser comparados. Espera-se que diferentes modelos de um mesmo sistema não forneçam valores preditos idênticos, mas razoavelmente aproximados; entretanto, os resultados encontrados por outros autores se baseiam em sistemas diferentes entre si e diferentes do sistema adotado nesta pesquisa. As diferenças estão na espécie estudada, $S$. zeamais e $S$. oryzae, no tipo de grão usado, milho e trigo e, principalmente, nos diferentes níveis de infestação inicial. Por exemplo, Hardman (1978), infestou trigo com uma densidade populacional de 143 insetos por kg, com a espécie $S$. oryzae. Ele usou trigo com temperatura de $23{ }^{\circ} \mathrm{C}$ e teor de umidade de $13,4 \%$. O modelo usado por ele predisse um crescimento populacional equivalente a 7.333 insetos por $\mathrm{kg}$ de trigo em 90 dias de armazenagem. O modelo apresentado neste trabalho, Eq. 1, para a mais alta infestação inicial estudada, 9,3 insetos por kg, e para a mesma temperatura usada por Hardman (1978), $23{ }^{\circ} \mathrm{C}$, prediz 914,9 insetos por kg de trigo. Longstaff (1981) estudou o crescimento populacional de $S$. oryzae em trigo com 14\% de umidade, infestado com 109,1 insetos por kg. Para $27^{\circ} \mathrm{C}$, seu modelo predisse uma população de adultos equivalente a 87.903,8 insetos por $\mathrm{kg}$ de trigo em 90 dias de armazenagem. O modelo apresentado neste trabalho (Eq. 1), para uma população inicial de 9,3 insetos por $\mathrm{kg}$ de trigo, prediz 9.603,4 insetos por kg de trigo, para a mesma temperatura. Maier et al. (1996) usaram um modelo de simulação para quantificar o efeito de práticas de manejo no crescimento populacional de $S$. zeamais em milho armazenado. Eles adotaram, como parâmetro de entrada para o modelo, a infestação inicial de um casal de adultos 
por 25,5 kg de milho, mais uma taxa de reinfestação de 12 pares de adultos adicionados a cada dia, em um silo metálico contendo 591,4 t de milho. Em torno de 45 dias de armazenamento e com a temperatura variando de 27 a $30{ }^{\circ} \mathrm{C}$, a população total de adultos atingiu 180.000 insetos. Todos esses autores encontraram resultados muito maiores porque usaram um grão mais úmido, o que é favorável ao crescimento populacional; além disso, Hardman (1978) e Longstaff (1981) trabalharam com a espécie $S$. oryzae que coloca muito mais ovos que a $S$. zeamais para uma mesma condição climática (Pedersen, 1992) mas, principalmente, todos eles partiram de uma densidade populacional inicial pelo menos dez vezes maior que as densidades estudadas na presente pesquisa. Portanto, embora as comparações citadas sirvam de ilustração, não servem para validar ou não os resultados apresentados.

Assim, embora os modelos aqui apresentados se limitem a apenas uma conformação do sistema de grãos armazenados: S. zeamais infestando trigo armazenado com $11,1 \%$ de umidade, eles descrevem perfeitamente a essência do crescimento populacional em relação ao efeito da temperatura e da densidade populacional inicial e cumprem os objetivos desta pesquisa, que é servir de base para outros estudos sobre modelagem de populações de insetos de grãos armazenados e podem ser prontamente utilizados no planejamento de outras pesquisas sobre o controle desses insetos e/ou na avaliação analítica de seus resultados.

\section{CONCLUSÕES}

1. O maior crescimento populacional de S. zeamais ocorreu a $28{ }^{\circ} \mathrm{C}$ e diminuiu drasticamente em temperaturas maiores ou menores que esta.

2. Na temperatura de $36^{\circ} \mathrm{C}$, todas as populações foram extintas entre 15 e 45 dias de armazenagem.

3. Quanto maior foi o nível de infestação inicial, maior também foi o crescimento populacional de $S$. zeamais.

4. O período de 90 dias de armazenagem sob $16^{\circ} \mathrm{C}$, não foi suficiente para exterminar as populações de $S$. zeamais em todos os níveis de infestação estudados.

5. Os modelos apresentados se limitam a apenas uma conformação do sistema de grãos armazenados: S. zeamais infestando trigo armazenado com 11,1\% de umidade e, para as condições estudadas, descrevem satisfatoriamente o crescimento populacional em relação ao efeito da temperatura e da densidade populacional inicial.

\section{AgRADECIMENTOS}

Sinceros e orgulhosos, os autores são gratos à Universidade Federal de Viçosa - UFV, à Fundação de Amparo à Pesquisa do Estado de Minas Gerais - FAPEMIG, à Coordenação de Aperfeiçoamento de Pessoal de Nível Superior CAPES, e à Financiadora de Estudos e Projetos - FINEP.

\section{LITERATURA CITADA}

Berryman, A. A. On the principles of population dynamics and theoretical models. American Entomologist, Lanham, v.43, n.4, p.147-151, 1997.

Guedes, R. N. C.; Dover, B. A.; Kambhampati, S. Resistance to chlorpyriphos-methyl, pirimiphos-methyl, and malation in brasilian and U.S. populations of Rhyzopertha dominica (Coleoptera: Bostrichidae). Journal of Economic Entomology, Lanham, v.89, n.1, p.27-32, 1996.

Guedes, R. N. C.; Lima, J. O. G.; Santos, J. P.; Cruz, C. D. Resistance to DDT and pyrethroids in a Brazilian populations of Sitophilus zeamais Motsch. (Coleoptera: Curculionidae). Journal of Stored Product Research, London, v.31, n.8, p.145150, 1995.

Hagstrum, D. W.; Flinn, P. W. Integrated pest management of stored-grain insects. In: Sauer, D. B. (ed.). Storage of Cereal Grains and their Products. St. Paul: American Association of Cereal Chemists, 1992. p.535-562.

Hagstrum, D. W.; Flinn, P. W. Integrated pest management. In: Subramanyam, B.; Hagstrum, D. W. (ed.). Integrated management of insects in stored products. New York: Marcel Dekker, 1996. p.399-409.

Hagstrum, D. W.; Throne, J. E. Predictability of stored-wheat insect population trends from life history traits. Environmental Entomology, Lanham, v.25, n.6, p.1354-1359, 1989.

Hardman, J. M. Life table data for use in deterministic and stochastic simulation models predicting the growth of insect populations under Malthusian conditions. The Canadian Entomologist, Lanham, v.108, n.9, p.897-906, 1976.

Hardman, J. M. A logistic model simulating environmental changes associated with the growth of populations of rice weevils Sitophilus oryzae, reared in small cells of wheat. Journal of Applied Ecology, Malden, v.15, n.8, p.65-87, 1978.

Hess, G. R. To analyze, or to simulate, is that the question? American Entomologist, Lanham, v.42, n.4, p.14-16, 1996.

Hwang, J. S.; Hsieh, F. K.; Kung, K. S. Influences of temperature and relative humidity on the development and reproduction of the maize weevil, Sitophilus zeamais Motschulsky. Plant Protection Bulletin, Rome, v.24, n.1, p.41-52, 1983.

Kawamoto, H.; Sinha, R. N.; Muir, W. E. Computer simulation modelling for stored-grain pest management. Journal of Stored Product Research, London, v.28, n.2, p.139-145, 1992.

Law, A. M.; Kelton, W. D. Simulation modeling and analysis. New York: McGraw-Hill, 1991. p.1-132.

Longstaff, B. C. The manipulation of the population growth of a pest species: an analytical approach. Journal of Applied Ecology, Malden, v.18, n.6, p.727-736, 1981.

Maier, D. E.; Adams, W. H.; Throne, J. E.; Mason, L. J. Temperature management of the maize weevil, Sitophilus zeamais Motsch. (Coleoptera: Curculionidae), in three locations in the United States. Journal of Stored Product Research, London, v.32, n.1, p.255-273, 1996.

Norton, G. A.; Holt, J.; Mumford, J. D. Decision analysis techniques. In: Norton, G. A.; Munford, J. D. (ed.). Decision tools for pest management, New York: CAB International, 1993. p.43-65. 
Pedersen, J. R. insects: identification, damage and detection. In: Sauer, D. B. (ed.). Storage of Cereal Grains and their Products. St. Paul: American Association of Cereal Chemists, 1992. p.435-491.

Smerage, G. H. Representation of development in insect population models. In: Goodenough, J. L.; McKinion, J. M. (ed.). Basics of Insect Modeling. St. Joseph: American Society of Agricultural Engineers, 1992. p.107-134.

SPSS Inc. SigmaPlot for Windows. Scientific Graphing Software. Version 4.00, 1997.
Statisoft, Inc. Statistica for Windows, release 4.2, 1993.

Throne, J. E. Life history of immature maize weevils Sitophilus zeamais Motschulsky (Coleoptera: Curculionidae) on corn stored at constant temperatures and relative humidities in the laboratory. Environmental Entomology, Lanham, v.23, n.4, p.1459-1471, 1994.

Throne, J. E. Computer modeling of the population dynamics of stored-product pests. In: Jayas, D.; White, N. D. G.; Muir, W. E. (ed.). Stored-Grain Ecosystems. New York: Marcel Dekker, 1995. p.169-197. 